Article

\title{
Simulation of Boiling Process in a Nanochannel with 700000 Argon Particles by Molecular Dynamics
}

\author{
Amin Etminan ${ }^{1}$, Davood Toghraie ${ }^{2, *}$, Zambri Harun ${ }^{3, *}$ and Ahmad Sharifian 4 \\ 1 Department of Mechanical Engineering, Shiraz Branch, Islamic Azad University, Shiraz, Iran; \\ etminan.amin@gmail.com \\ 2 Department of Mechanical Engineering, Khomeinishahr Branch, Islamic Azad University, Isfahan, Iran; \\ davoodtoghraie@gmail.com \\ 3 Department of Mechanical and Materials Engineering, Faculty of Engineering and Built Environment, \\ 43600 UKM Bangi, Selangor, Malaysia; zambri@ukm.edu.my \\ 4 School of Mechanical and Electrical Engineering, University of Southern Queensland, \\ Toowoomba QLD 4350, Australia; Ahmad.Sharifian-Barforoush@usq.edu.au \\ * Correspondences: davoodtoghraie@gmail.com; Tel.: +98-913-322-1167,
} zambri@ukm.edu.my; Tel.: +60-19-665-2833

\begin{abstract}
In this paper, the boiling flow inside a nanochannel with 700000 argon particle has been simulated by molecular dynamics (MD) simulation. This approach has been employed to analyse the superheated flow and its heat transfer pattern as well. For all simulations, an external thrust force varying from $1 \mathrm{PN}$ to $12 \mathrm{PN}$ is exerted on inlet nanoparticles along the channel to have the forced annular boiling flow. Computations reveal that increase of saturation condition and superheated region have significant impacts on the liquid-vapor interface. Furthermore, because of the major influence of surface tension throughout the nanochannel, the x-velocity of liquid film and vapor core has no considerable fluctuations and stay smooth. Results show the behaviors of what significantly similar with the author's previous published simulation with 700000 solid particles.
\end{abstract}

Keywords: Nanochannel; Molecular Dynamics Method; Nanoparticle, Argon; Boiling Process.

\section{Introduction}

Nowadays, the majority of macroscale and nanoscale heat and mass transfer research is focused on boiling and phase change flows issues. Improvement of thermal energy efficiency of engineering processes is rooted in more vivid and clear understanding of these phenomena. Experimental measurements can considered as efficient means for determining the flow structure, analysis the boiling flow and describing the heat transfer characteristics in micro and nanochannels. Besides, huge progresses in computer science and computational techniques make an adequate possibility with more resolution as well as time consuming method to solve such programmes.

The extremely powerful technique of molecular dynamics simulation involves solving the classical many-body problem in contexts relevant to the study of matter at the atomic level. Since there is no alternative approach capable of handling this extremely broad range of problems at the required level of detail, molecular dynamics methods have proved indispensable in both pure and applied research [1]. Molecular dynamics simulation is a powerful tool to capture the microscopic view of the nanoscale phenomena. Previously, molecular dynamics simulations have been carried out by a number of researchers to understand the molecular behavior of the phase transition from liquid to vapor. It has been demonstrated that molecular dynamics simulation is an efficient tool to investigate these complicated phenomena. The molecular dynamics study of these effects could provide information that is difficult to be obtained by experiments, and thereby improve our understanding in the technological processes such as flow in microporous media, and transport of biological and chemical materials through nanodevices or microdevices. Furthermore, Newton's law can be simulated and averaging of the molecular system provides some thermodynamical parameters including pressure velocity and temperature [1]. 
Most of the researchers' focus was on the heterogeneous phase transition of liquid from a molecular level flat solid surface. Phase transition from a solid surface can occur in a normal boiling mode or in an explosive boiling mode depending on the surface superheat temperature [2]. The complete evaporation of a three-dimensional submicron droplet under subcritical conditions has been modeled by Long et al [3] using molecular dynamics. The two-phase system consisted of 2048 argon atoms was first allowed to relax to equilibrium, then the droplet was evaporated by increasing the temperature of the vapor phase atoms at the boundaries of the system until only the vapor phase remained. The computed evaporation rate agrees with that predicted by the Knudsen aerosol theory.

The thermal conductivity of solid thin films in the direction perpendicular to the film plane has been carried out by Lukes et al. [4] by the molecular dynamics computational technique. They also delineated the conditions necessary for meaningful thermal conductivity calculations and offered recommendations for efficient simulations as well. Their research demonstrates the potential of molecular dynamics for ascertaining microscale thermophysical properties in complex structures. $\mathrm{Yi}$ et al. [5] performed molecular dynamics simulations of the vaporization phenomenon of an ultra-thin layer $(2 \mathrm{~nm})$ of liquid argon on a platinum surface. The results reveal trends that agree with our knowledge of vaporization of a similar macroscopic system. For example, for the high surface temperature the vaporization process is reminiscent of the Leidenfrost phenomenon and after the formation of a vapor region between the surface and the liquid mass, the latter deforms and tends to approximately acquire a spherical "droplet" shape, as one would have expected from macroscopic considerations. Wang et al. [6] investigated the net evaporation process by performing non-equilibrium molecular dynamics (NEMD) simulations. They found the evaporation coefficients of the nonequilibrium molecular dynamics agree with those from the transition state theory and some data of equilibrium molecular dynamics calculations in literature within 15\%. Toghraie and Azimian [7] investigated Molecular dynamics simulation of annular flow boiling of 3200 Argon atoms with the modified Lennard-Jones potential function. They found that saturation condition and superheat degree have great influences on the liquid-vapor interface. Also, the results show that due to the relatively strong influence of the surface tension in small channels, the interface between the liquid film and the vapor core is fairly smooth, and the mean velocity along the stream-wise direction does not change anymore. Morshed et al [8] investigated the boiling phenomena of thin liquid films adsorbed on a nanostructured solid surface. Their results indicated nanostructures play a significant role in both cases: Argon responds very quickly for the nanostructured surface, the transition from liquid to vapor becomes more gradual, and the evaporation rate increases with the nanoposts height.

In the present study, molecular dynamics simulations have been employed to investigate the boiling flow of simple Lennard-Jones fluids subject to an inlet driving force in a nanochannel. The main purpose of this research is to scrutinize the influence of modified Lennard-Jones potential function on the temperature, heat flux, velocity and surface tension profiles in order to comprehend the boiling flow behavior in nanochannel geometry. As far we know, no one has reported any investigation for these phenomena.

\section{Simulation Procedure}

The molecular dynamics simulation is employed to describe the forced annular boiling flow in a nanochcannel with 700000 argon atoms placed and simulated in cuboids of dimension $L_{x} \times L_{y} \times L_{z}=45 \sigma \times 45 \sigma \times 900 \sigma$ where $\sigma$ is the length scale parameter and it is included a solid platinum wall, liquid argon layers, and an argon vapor. Each atom is spaced $1.15 \sigma$ from its neighboring atoms in all three directions. Figure 1 illustrates a schematic of MD domain simulation. According to this figure, argon molecules are divided into three regions: the vapor molecules are moving along the center of the channel and the liquid molecules comprise two thin annular films along the channel walls sandwich the vapor phase portion. At the bottom and top surfaces, wall boundary conditions are considered. Also, the system has doubly periodic boundary conditions in the $x$ and $y$ directions. The velocity of an atom crossing the $x$ and $y$ boundaries remains constant. An 
external thrust force varying from $1 \mathrm{PN}$ to $12 \mathrm{PN}$ is exerted on inlet nanoparticles confined between two triple-layer solid Platinum walls.

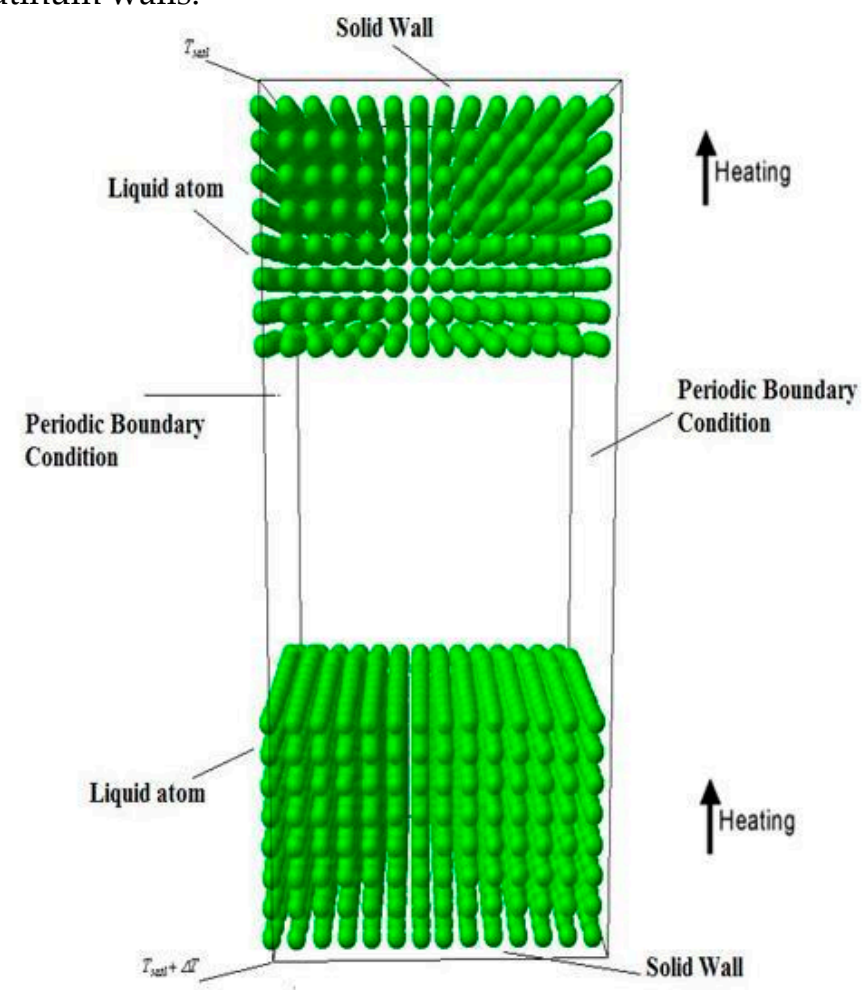

Figure 1. Schematics of MD domain simulations

Newton's second law as in equation (1) in all MD simulations should be solved for all fluid molecules. Before MD simulation, an integration method for the mentioned equation, the potential model and the simulation cell are computed.

$\vec{F}_{i}=\sum_{i \neq j} F_{i j}=m_{i} \frac{d^{2} \vec{r}_{i}}{d t^{2}}=m_{i} \frac{d \overrightarrow{v_{i}}}{d t}$

The exerting force of each molecule can be described as the gradient of the potential function of a molecule $\vec{F}_{i j}=-\vec{\nabla}\left(\phi+\phi_{w}\right)$. Lennard-Jones particles with the MD simulations lead to modified Lennard-Jones potential function in the NVT ensemble where $N, V$ and $T$ mean the number of particles in system, system's volume and temperature respectively, [9]. Potential function presented by Stoddard and Ford [10] can be used to modeling the relations between fluid particles separated by a distance $r_{i j}$ as follow:

$\varphi\left(r_{i j}\right)=\left\{4 \mathcal{\varepsilon}\left[\left(\frac{\sigma}{r_{i j}}\right)^{12}-\left(\frac{\sigma}{r_{i j}}\right)^{6}\right]\right\}+\left\{4 \mathcal{\varepsilon}\left[6\left(\frac{\sigma}{r_{c}}\right)^{12}-3\left(\frac{\sigma}{r_{c}}\right)^{6}\right]\right\}\left(\frac{r_{i j}}{r_{c}}\right)^{2}-\left\{4 \mathcal{\varepsilon}\left[7\left(\frac{\sigma}{r_{c}}\right)^{12}-4\left(\frac{\sigma}{r_{c}}\right)^{6}\right]\right\}$

where the distance between the centres of $i^{\text {th }}$ and $j^{\text {th }}$ spherical particles is defined as $r_{i j}=\left|r_{i}-r_{j}\right|, \varepsilon$ is a parameter corresponding to the energy and is called the maximum potential depth and $r_{c}=4.5 \sigma$ is the cut-off radius. Thompson and Robbins [11] understood that decreasing $r_{c}$ to less than $2 \sigma$ made considerable changes in the potential, boundary conditions and other parameters. Therefore, argon atom is used as the LJ fluid with the following potential parameters in the present work: $m=6.63 \times 10^{-26} \mathrm{~kg}, \sigma=3.045 A^{\circ}$ and $\varepsilon=1.67 \times 10^{-21} \mathrm{~J}$. Furthermore, three layers of face centered cubic (FCC) surface of Platinum molecules with parameters $m_{s}=3.24 \times 10^{-26} \mathrm{~kg}, \sigma_{s}=2.475 A^{\circ}$ and $\varepsilon_{s}=0.835 \times 10^{-21} \mathrm{~J}$ presented by [12-17] are used as the solid wall. 
The influence of solid walls on the velocity, surface tension and temperature distribution can be simulated by a bottom wall held at a superheated temperature, i.e. $T_{\text {sat }}+\Delta T$ and an upper wall held at a saturation temperature $T_{\text {sat }}$ and the degree of superheat $\Delta T$. The superheat degree not only makes a constant temperature gradient between the bottom and top walls in the $\mathrm{z}$ direction but also provides a driving force for heat flux.

Tsuruta and Nagayama [17] presented a few useful relations for modeling of the interaction between solid wall and liquid phase of flow as follow:

$\phi_{S}\left(r_{i j}\right)=\left\{4 \varepsilon_{s f}\left[\left(\frac{\sigma_{s f}}{z}\right)^{12}-\left(\frac{\sigma_{s f}}{z}\right)^{6}\right]\right\}+\left\{4 \varepsilon_{s f}\left[6\left(\frac{\sigma_{s f}}{r_{c}}\right)^{12}-3\left(\frac{\sigma_{s f}}{r_{c}}\right)^{6}\right]\right\}\left(\frac{z}{r_{c}}\right)^{2}-\left\{4 \varepsilon_{s f}\left[7\left(\frac{\sigma_{s f}}{r_{c}}\right)^{12}-4\left(\frac{\sigma_{s f}}{r_{c}}\right)^{6}\right]\right\}$

where $\sigma_{s f}$ and $\varepsilon_{s f}$ can be computed according to the Kong mixing rule [18] as follow:

$$
\begin{aligned}
\sigma_{S f} & =\left[\frac{1}{2^{13}} \frac{\varepsilon \sigma^{12}}{\sqrt{\varepsilon \sigma^{6} \varepsilon_{s} \sigma_{s}^{6}}}\left(1+\left(\frac{\varepsilon_{s} \sigma_{s}^{12}}{\varepsilon \sigma^{12}}\right)^{\frac{1}{13}}\right)^{13}\right]^{\frac{1}{6}} \\
\varepsilon_{s f} & =\frac{\sqrt{\varepsilon \sigma^{6} \varepsilon_{s} \sigma_{s}{ }^{6}}}{\sigma_{s f}{ }^{6}}
\end{aligned}
$$

Verlet [19] presented the following equations to compute the particle's trajectories:

$$
\begin{aligned}
& V(t+\delta t)=V(t)+\frac{1}{2}\left[\frac{F(t)+F(t+\delta t)}{m}\right] \delta \delta t \\
& r(t+\delta t)=r(t)+V(t) \delta t+\frac{F(t)}{m} \frac{\delta t^{2}}{2}
\end{aligned}
$$

The time step $\delta t=5$ fs has been employed in all simulations. Gaussian distribution based on the specific temperature can be used as the initial condition for each molecule as the following formula:

$\frac{1}{N_{a t m}} \sum_{i=1}^{N_{a t m}} \frac{1}{2} m\left|V_{i}^{2}\right|=\frac{3}{2} k_{B} T$

NEMD simulation is employed when the system reaches equilibrium state after 500000 time steps. At least 2000000 time steps are used for sample data gathering for all simulations. Additionally, the velocity rescaling $V_{\text {new }}=\lambda V_{\text {old }}$ is first updated from the force acting on the particles and $\lambda$ is defined as $\lambda=\left(T_{\text {target }} / T_{\text {global }}\right)^{1 / 2}$ where $T_{\text {target }}$ is the target temperature and $T_{\text {global }}$ is the global temperature of the fluid particles in the system. The instantaneous temperature is defined as a fluctuation of kinetic energy per particle as follow:

$T_{\text {inst }}=\frac{\frac{1}{2} m\left(V_{x}^{2}+V_{y}^{2}+V_{z}^{2}\right)}{3 n(z) k_{B}}$

where $\mathrm{n}(z)$ is the number of atoms at height $\mathrm{z}$.

In the $\mathrm{z}$ direction, the system is confined by two stationary walls and periodic conditions are employed along the $x$ - and $y$-directions as well. Based on the results of Alexander and Garcia [20], the thermal wall boundary condition is applied for particles near the walls. They showed when the liquid particle is near the wall surface, the repulsive force will push the liquid particle away from the wall surface, but some liquid particles may collide with the wall surface. Then the bottom wall at the temperature of $T_{\text {sat }}+\Delta T$ and all velocity components are reset to a biased Maxwellian distribution presented by [20]:

$$
\begin{aligned}
& V_{x i}=\sqrt{\frac{k_{B}\left(T_{s a t}+\Delta T\right)}{m_{i}}} \psi_{1} \\
& V_{y i}=\sqrt{\frac{k_{B}\left(T_{s a t}+\Delta T\right)}{m_{i}}} \psi_{2} \\
& V_{z i}= \pm \sqrt{\frac{-2 k_{B}\left(T_{s a t}+\Delta T\right)}{m_{i}} \ln \psi_{3}}
\end{aligned}
$$


In above equations $k_{B}$ is the Boltzmann constant, $m_{i}$ is the mass of each molecule, $\psi_{3}$ is a uniformly distributed number in range of $(0,1), \psi_{1}$ and $\psi_{2}$ are Gaussian-distributed random numbers with zero mean and unit variance. Also, when a particle collides with the upper wall at the temperature of $T_{\text {sat }}$, all three components of the velocities are reset to a biased Maxwellian distribution [20]:

$V_{x i}=\sqrt{\frac{k_{B}\left(T_{s a t}\right)}{m_{i}}} \psi_{1}$
$V_{y i}=\sqrt{\frac{k_{B}\left(T_{s a t}\right)}{m_{i}}} \psi_{2}$
$V_{z i}= \pm \sqrt{\frac{-2 k_{B}\left(T_{s a t}\right)}{m_{i}} \ln \psi_{3}}$

When the system reaches the steady state, the flow velocity, temperature, surface tension and density profiles are computed as a function of channel height. The program was run for 1500000 time steps for time averaging. In order to compute density, surface tension, liquid film velocity, vapor core velocity and temperature profiles, the domain is divided into $N_{b i n, z}=512$ rectangular bins along the $z$-direction, each bin with height $\Delta z=L z / N_{b i n, z}$, where $L z$ is the box length along the $z$-direction. Also, the domain is divided into $N_{b i n, x}=12$ bins along the $x$-direction, each bin with length $\Delta x=L_{x} / N_{b i n, x}$, where $L_{x}$ is the box length along the $x$-direction. Hence, the volume of each bin is $\Delta x \times \Delta z \times L_{y}$, where $L_{y}$ is the box length along the $y$-direction. For a large number of particles, more bins are needed. For adding molecules in each layer, $H_{n}\left(z_{i}\right)$ is defined as:

$$
\begin{array}{cc}
H_{n}\left(z_{i, j}\right)=1 & (n-1) \Delta z \leq z_{i} \leq n \Delta z \\
H_{n}\left(z_{i, j}\right)=0 & \text { otherwise }
\end{array}
$$

where the subscript $j$ represents the $j^{\text {th }}$ time step. Hence, the local average density in the $n^{\text {th }}$ slab from time step $I_{\text {start }}$ to $I_{\text {end }}$ is [21]:

$$
n(z)=\frac{m \sum_{j=I_{\text {start }}}^{I_{\text {end }}} \sum_{i=1}^{N_{\text {atm }}} H_{n}\left(z_{i, j}\right)}{L_{x} L_{y} \Delta z\left(I_{\text {start }}-I_{\text {end }}+1\right)} \quad 0 \leq x_{i} \leq \Delta x
$$

where $m$ is the mass of each molecule and $x_{i}$ and $z_{i}$ is the coordinate of the mid-point of the $n^{\text {th }}$ slab. When sampling begins, the molecule number in each slab is accounted for and the local density can be considered.

At the atomic scale, the surface tension can be expressed as the integrated imbalance of normal and tangential pressure near the interface. The following expressions are used to calculate the two components of the pressure tensor presented by Haile [22],

$$
\begin{aligned}
& P_{N}(z)=n(z) k_{B} T-\frac{1}{2 A}\left\langle\sum_{i \neq j} \frac{z_{i j} \phi^{\prime}\left(r_{i j}\right)}{r_{i j}} \theta\left(\frac{z-z_{i}}{z_{i j}}\right) \theta\left(\frac{z_{j}-z}{z_{i j}}\right)\right\rangle \\
& P_{T}(z)=n(z) k_{B} T-\frac{1}{4 A}\left\langle\sum_{i \neq j}^{\left[\frac{\left.x_{i j}^{2}+y_{i j}{ }^{2}\right]}{r_{i j} z_{i j}} \theta\left(r_{i j}\right)\right.} \theta\left(\frac{z-z_{i}}{z_{i j}}\right) \theta\left(\frac{z_{j}-z}{z_{i j}}\right)\right\rangle \\
& \theta(x)=1 \quad x \geq 0 \\
& \theta(x)=0 \quad x<0
\end{aligned}
$$

where the $n(z)$ is the value of density at the height $z$, the $i<j$ notation indicates a summation over all distinct pairs $i$ and $j$ without counting any pair twice (i.e. as $i j$ and $j i), k_{B}$ is the Bolzmann's constant, \langle\rangle denotes an ensemble average taken over the duration of the simulation for which thermal equilibrium exists. In terms of atomic position and liquid-vapor potential $\varphi$ the expression for surface tension is presented by Haile [22],

$\gamma_{l v}=\int_{-\infty}^{+\infty}\left[P_{N}(z)-P_{T}(z)\right] d z$ 


\section{Results}

The influence of the saturation temperature on the density distribution according to the NEMD simulation has been illustrated in Figure 2. The highest magnitude of density is found adjacent to the solid walls of channel where a thin film of non-evaporative fluid is. On the contrary, the lowest value of density is observed at the centre of channel where the effects of solid walls as well as saturation temperature are insignificant. As can be seen, because of significant solid-liquid interactions, the highest and lowest values of density in the snapshots are related to saturation temperature $108 \mathrm{~K}$ and $145 \mathrm{~K}$ respectively. There is a continuous transition for density from the liquid to the vapor phase under all simulation conditions in a far distance from solid walls of channel. The behaviors of density distribution and interface structure are in good agreement with the previous study [23]. With regards to the nanoscale of the space between the walls, the impact of the solid wall adsorption on the concentration of the liquid particles is very remarkable.

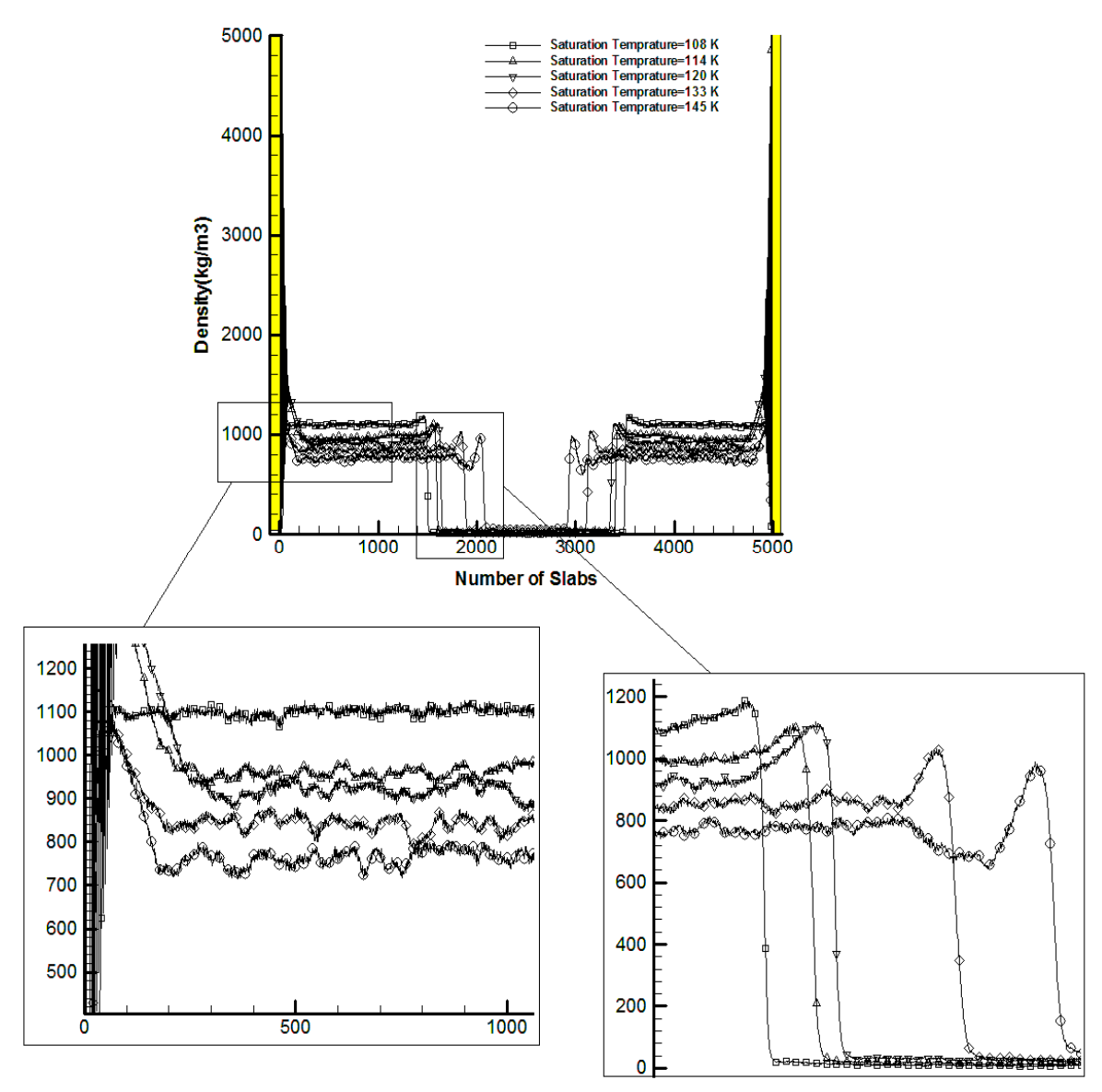

Figure 2. Influence of saturation temperature on the density profiles

As mentioned before, the bottom wall temperature and the top wall temperature can be considered as superheated and saturation temperatures respectively. Figure 3 illustrates the variation of density on the cross section area of the channel with regards to different superheated and saturation temperatures. It can be easily found that the bottom wall temperature has no significant impact on density distribution in particular at the centre of the channel where the stationary walls have negligible effect on flow structure and physical parameters as well. Therefore, density value and its distribution are independent of driving force and bottom wall temperature. 


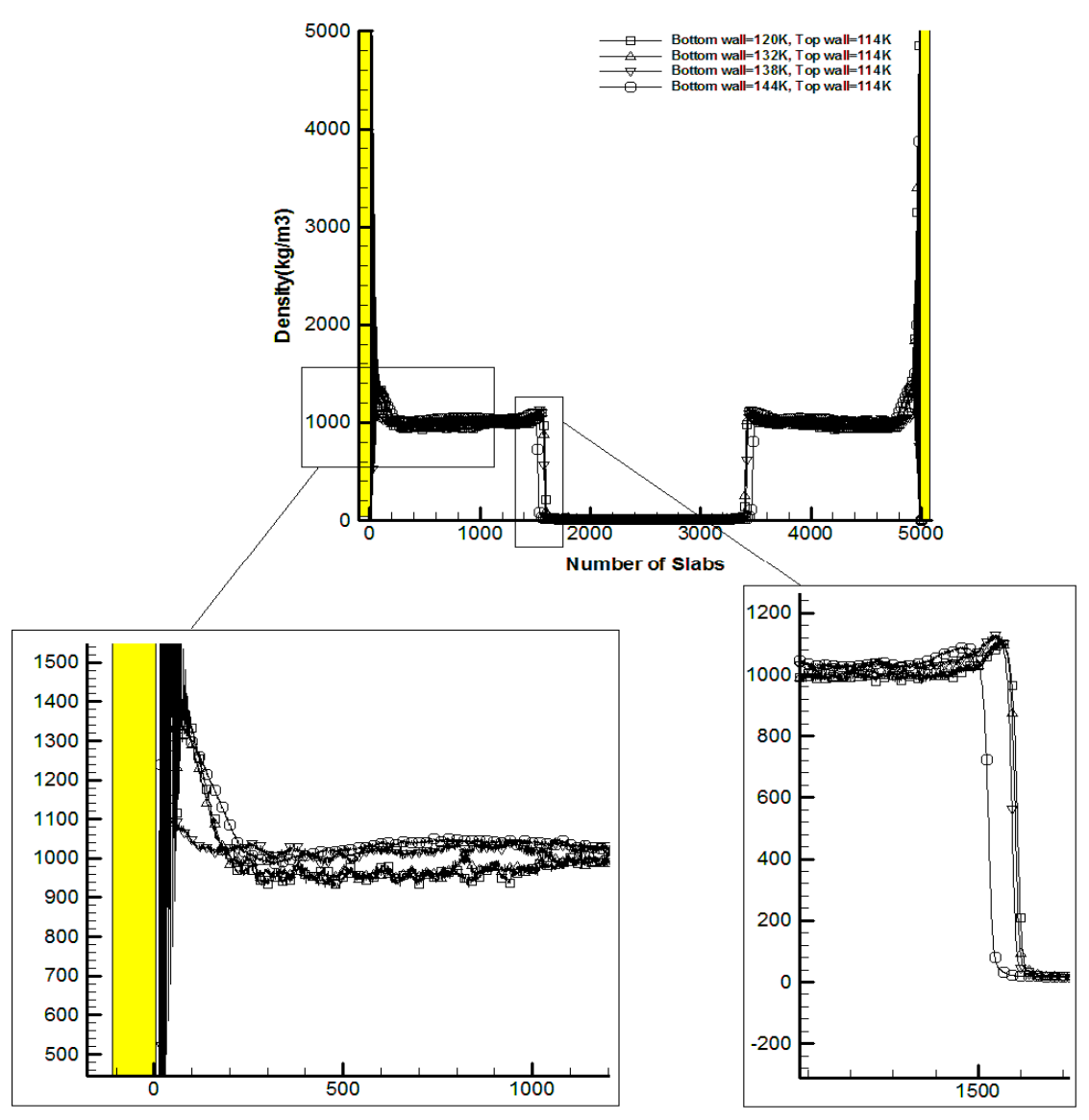

Figure 3. Influence of bottom wall temperature on the density profiles

The local surface tension distribution in perpendicular to the solid walls of channel has been depicted in Figure 4 for different superheat degrees and a constant saturation temperature of $96 \mathrm{~K}$. According to this figure, interfacial regions in all simulation cells cause two maximum values of surface tension as it can be seen in the zoomed view of Figure 4. The surface tension distribution varies remarkably in far distance from solid walls or at the centre of channel where the liquid-vapor interface zone is dominant. Furthermore, as it can be seen the bottom wall temperature has insignificant effect on the local surface tension distribution. 


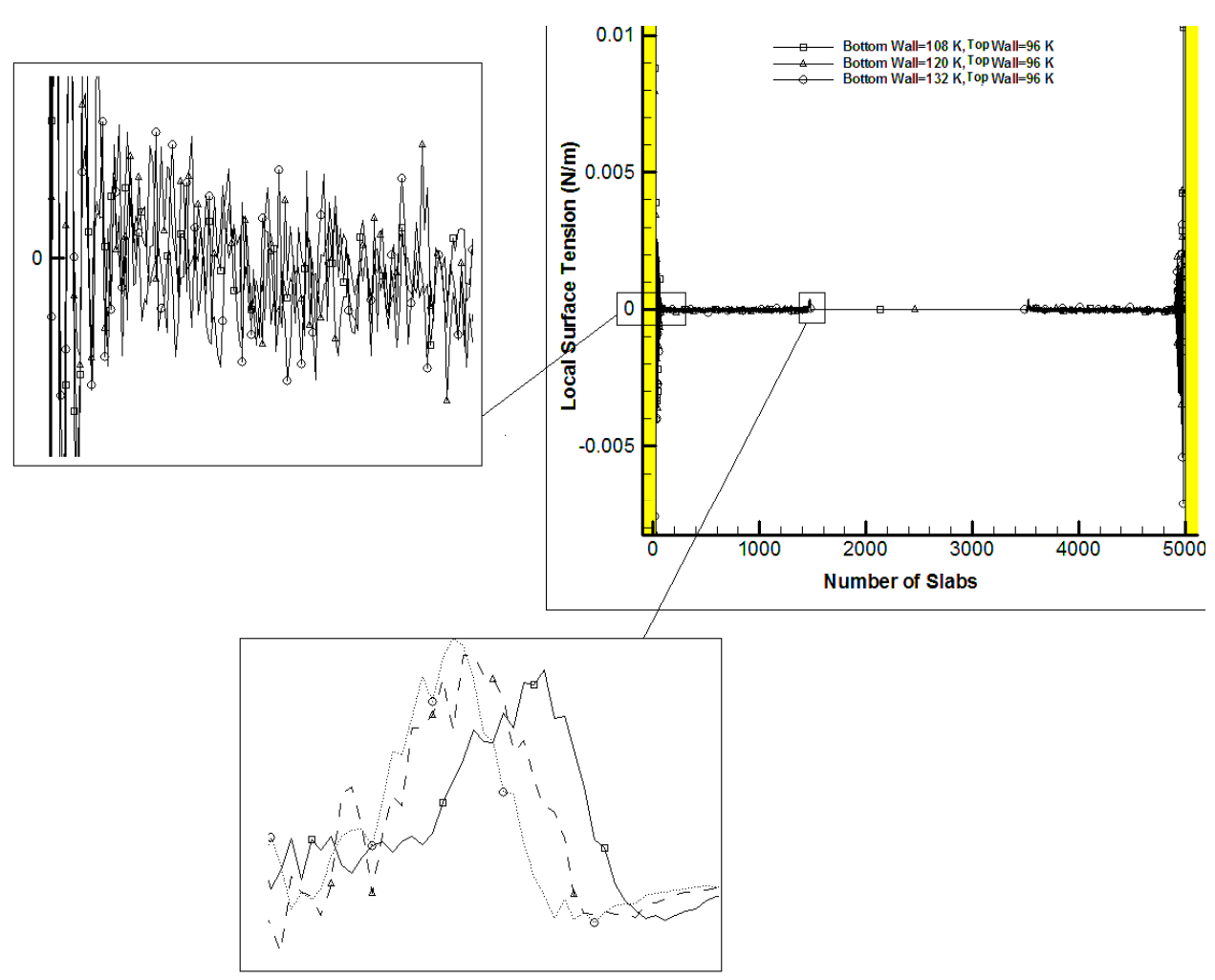

Figure 4. Local surface tension at different superheat degrees at saturation temperature of $96 \mathrm{~K}$

The effect of different superheat degrees on temperature profile on the cross section area of the channel is illustrated in Figure 5 where the magnitude of temperature is computed from equation (12) and saturation temperature is considered a constant value of $108 \mathrm{~K}$ for all simulations. As can seen, the temperature magnitude reaches a value higher than saturation temperature of $108 \mathrm{~K}$ due to the superheat degree. On the plus side, the fluctuating domain of temperature becomes wider when superheat temperature rises. The temperature jump adjacent to the solid wall causes a rise of discontinues across the liquid-solid wall interface. Since, the local temperature of this kind of problem is described as a combination of velocity of atoms and transferred momentum between them, therefore, the motion of each atom can be influenced by other neighboring atoms significantly. Moreover, each atom is affected by other neighboring atoms of liquid more than neighboring atoms of walls. This behavior makes a sudden change on kinetic energy distribution at wall-liquid interface. 


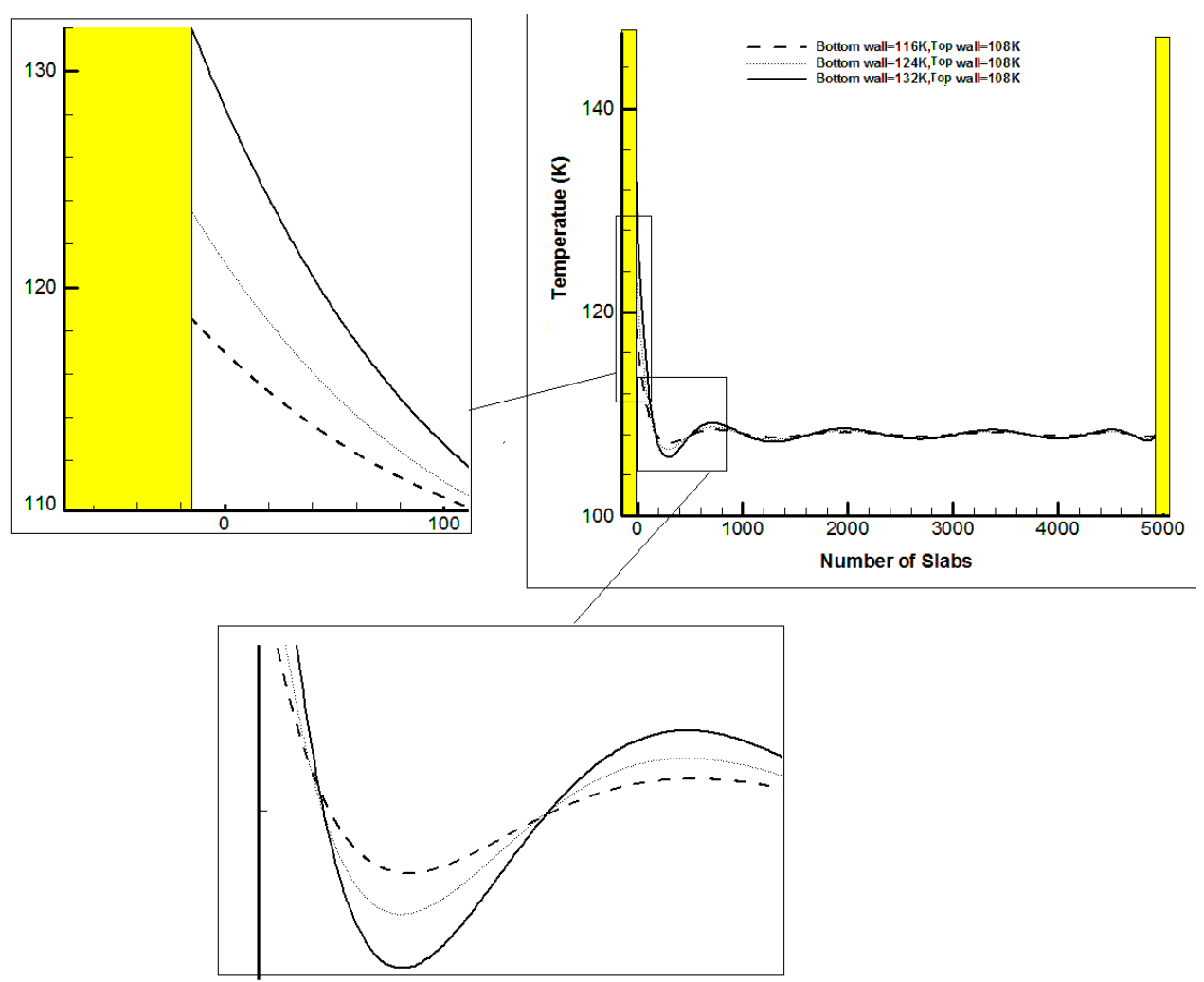

Figure 5. Temperature profiles at different superheated temperature for the bottom wall and at saturation temperature of $108 \mathrm{~K}$ for the top wall

The variation of $x$-velocity components of mean liquid film and mean vapor core for different external force has been illustrated in Figures 6 and 7, respectively. As it can be seen, the significant impact of surface tension throughout a nanochannel, the x-velocity of liquid film and vapor core has not considerable fluctuations and stay smooth. This trend is in good agreement with the majority of earlier works such as $[9,24]$.

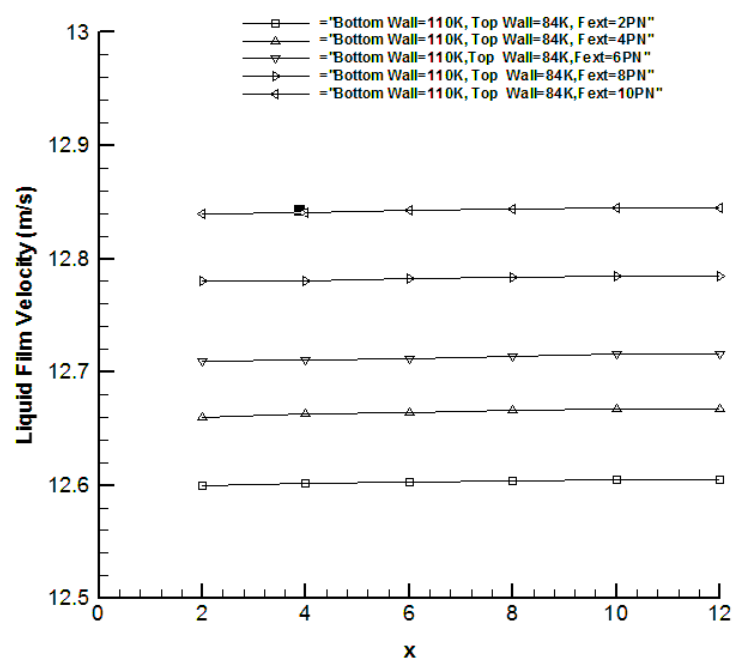

Figure 6. Variation of the x-velocity component of mean liquid film. 


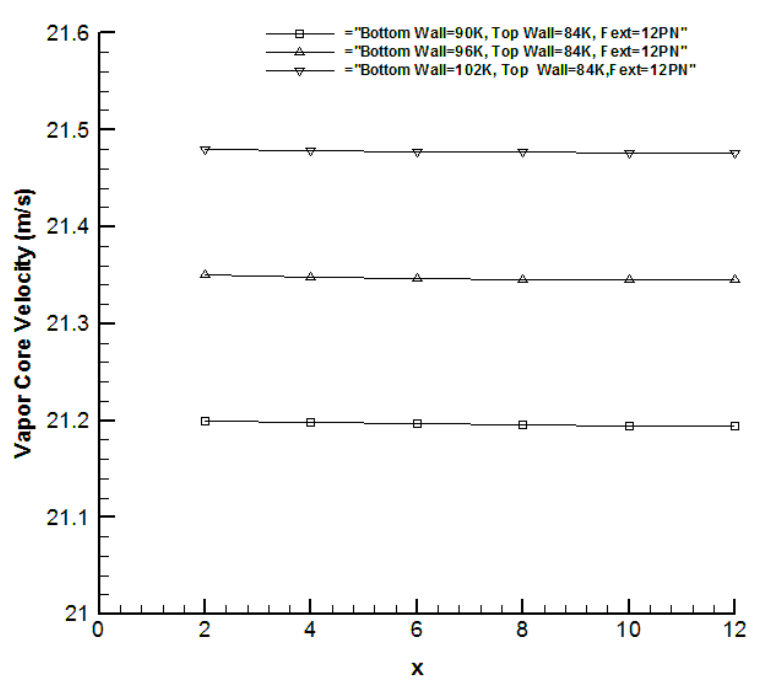

Figure 7. Variation of the $\mathrm{x}$-velocity component of mean vapor core

Variation of heat flux across the simulation domain in the z-direction obtained from equation (23) versus bottom wall temperature at saturation temperatures of $96 \mathrm{~K}$ for external forces ranging from 2 to 10 PN are shown in Figure 8.

$\vec{q}^{\prime \prime}=\frac{1}{2 \forall}\left[\sum_{i=1}^{N_{\text {atm }}} m_{i}\left|v_{i}\right|^{2} \vec{v}_{i}+\sum_{i=1}^{N_{\text {atm }}} \sum_{j \neq i}^{N_{\text {atm }}} \varphi_{i j} \vec{v}_{i}-\sum_{i=1}^{N_{\text {atm }}} \sum_{j \neq i}^{N_{\text {atm }}}\left(\vec{r}_{i j} \vec{f}_{i j}\right) \vec{v}_{i}\right]$

According to Figure 8, since $\Delta T$ is the driving force for heat flux, a higher $\Delta T$ results in a larger heat flux value, which is still in accordance with Fourier's law of heat transfer, qualitatively. These figures show that a higher external force results in a larger heat flux value. It could be explained that an increase in driving force will increase the fluid mobility and as a result, the heat dissipation will rise. This phenomenon could increase the heat flux in the simulation domain. Hence, it is concluded that the heat flux heavily depends on the degree of superheat and external force. On the other hand, the heat flux also depends on the boundary conditions.

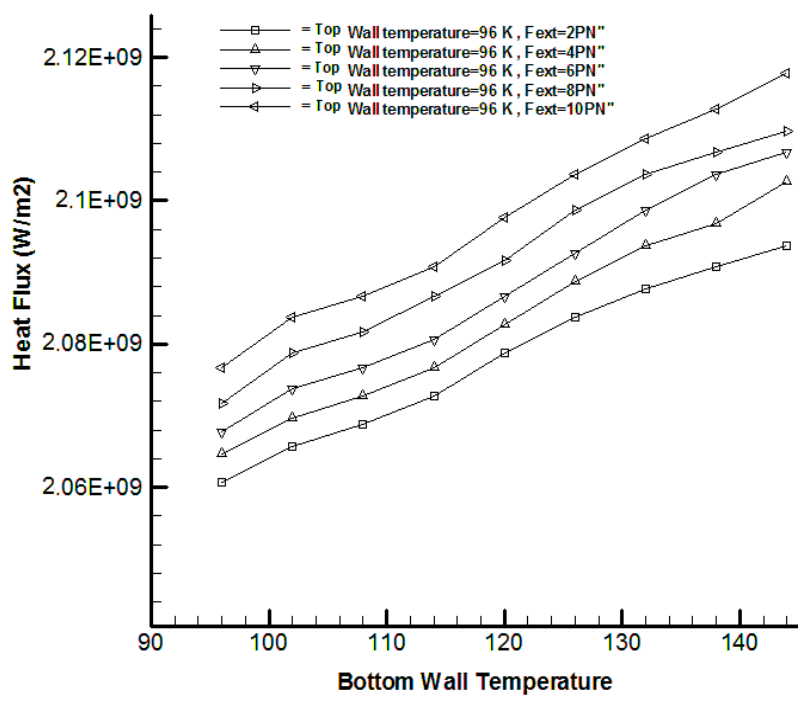

Figure 8. Variation of heat flux versus bottom wall temperature for different external force 


\section{Conclusions}

Molecular dynamics (MD) simulations have been carried out to investigate the boiling flow in a nanochannel with 700000 particles in this research. The computational results reveal that the bottom wall temperature has no important impact on density distribution in particular at the centre of the channel where the solid walls have small effect on flow pattern and physical parameters too. Furthermore, the surface tension distribution varies remarkably in far distance from solid walls or at the centre of channel where the liquid-vapor interface zone is dominant. At higher temperatures and at a higher degree of superheat, the surface tension drops by increasing the temperature as well.

Additionally, as it can be seen, the significant influences of surface tension throughout a nanochannel and the interface between the liquid film and the vapor core, the $\mathrm{x}$-velocity has not considerable fluctuations and stay smooth along the streamwise.

Acknowledgments: This research is supported by Universiti Kebangsaan Malaysia (UKM) under grant Fundamental Research Grant Scheme provided by the Government of Malaysia.

Author Contributions: This paper is extracted from a teamwork research. Amin Etminan and Davood Toghraie conceived and designed the numerical simulations and gathered all data meanwhile Zambri Harun and Ahmad Sharifian provided supervisory roles related to the data analysis and proofreading.

Conflicts of Interest: The authors declare no conflict of interest.

\section{Appendix A}

The nomenclature used in the manuscript has been tabled as follow:

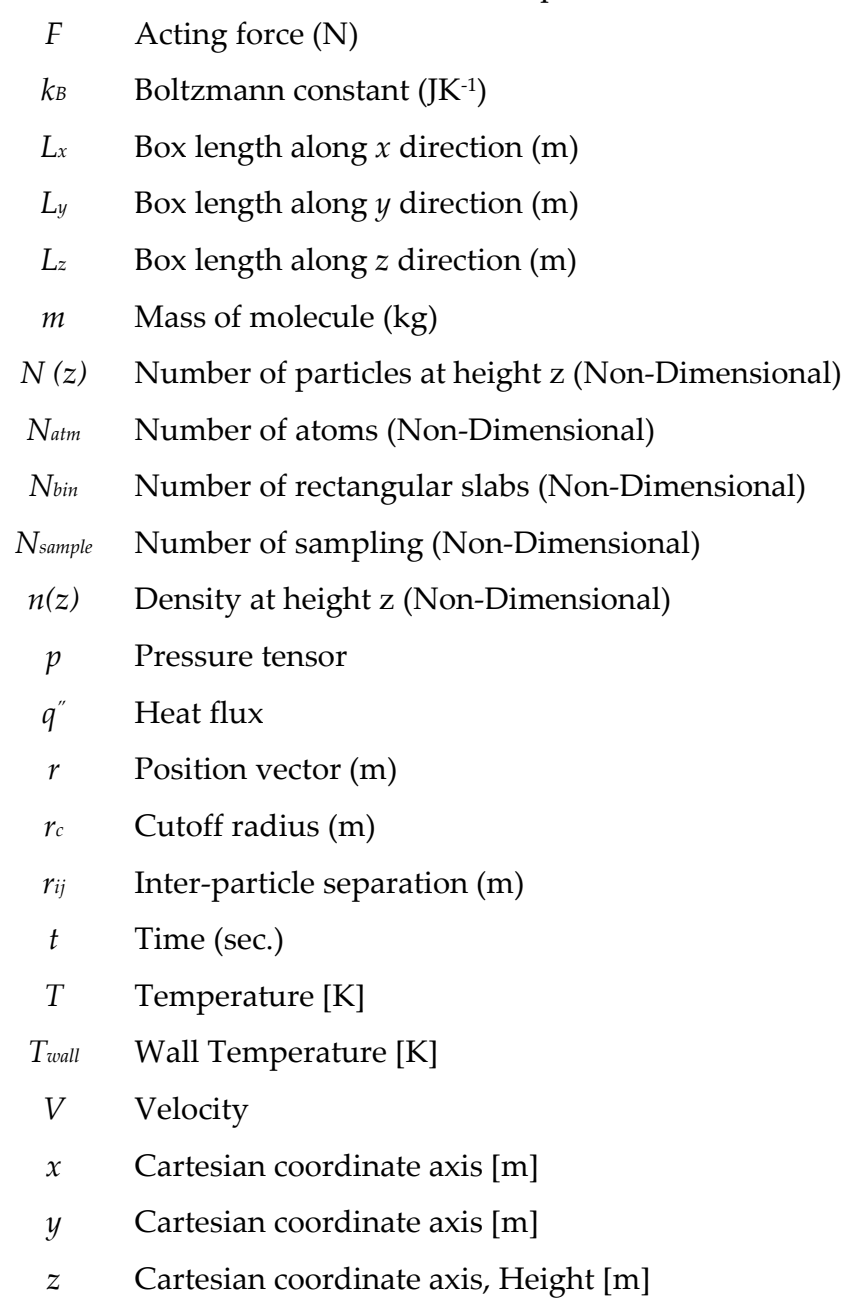

\section{Greek Letters}

$\vec{\nabla} \quad$ Dell gradient

$\Delta \mathrm{z} \quad$ Thickness of rectangular slab (m) 


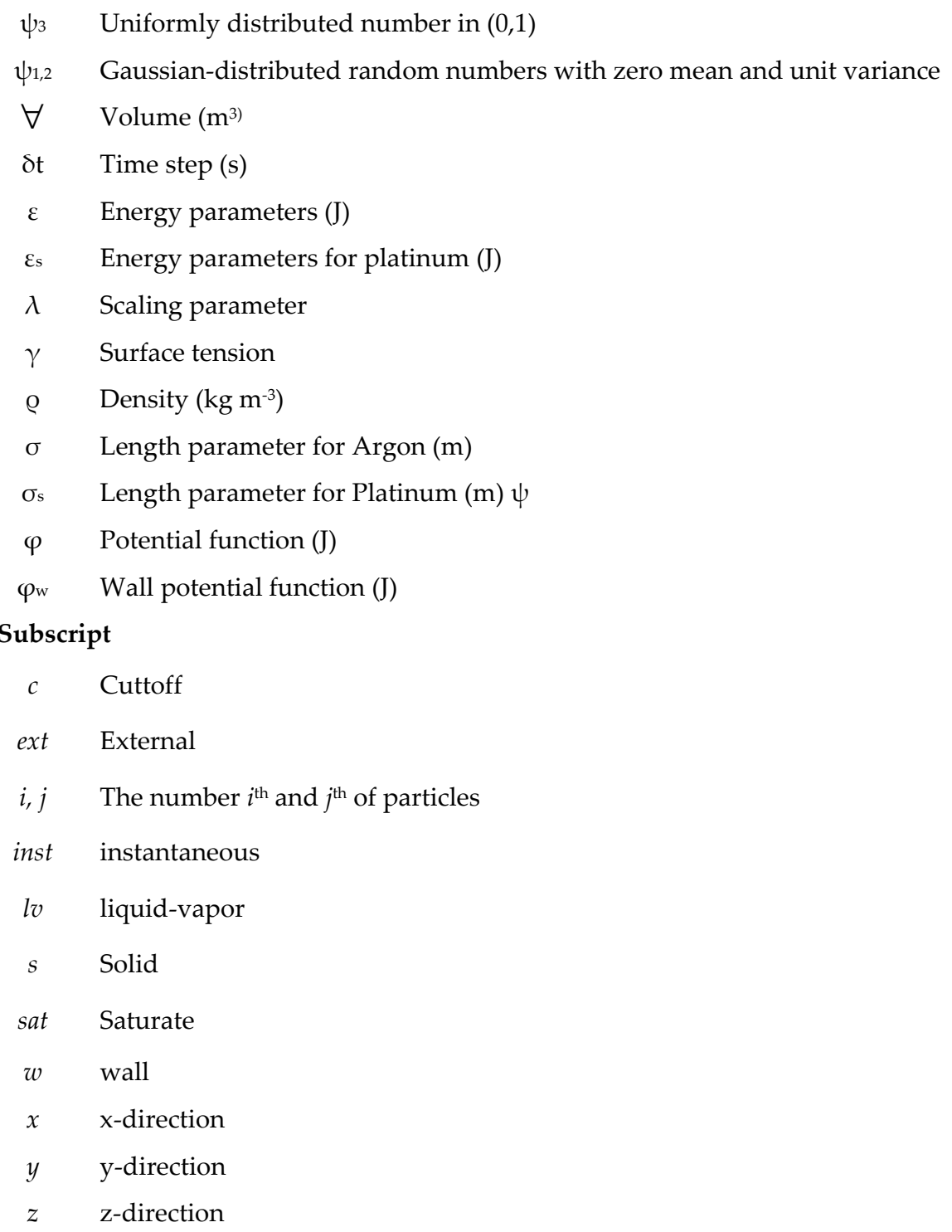

\section{References}

1. Rapaport, D. C. The art of molecular dynamics simulation. $2^{\text {nd }}$ edition, England: Cambridge University Press, 2004.

2. Gu, X.; Urbassek, H. M. Atomic dynamics of explosive boiling of liquid-argon films. Applied Physics B Lasers and Optics, 2005, Vol. 81, No. 5, pp. 675-679.

3. Long, L. N.; Micci, M. M.; Wong, B. C. Molecular dynamics simulation of droplet evaporation. Computer Physics Communications, 1996, Vol. 96, pp. 167-172.

4. Lukes, J. R.; Li, D. Y.; Liang, X. G.; Tien, C. L. Molecular dynamics study of solid thin-film thermal conductivity. Journal of Heat Transfer, 2000, Vol. 122, No. 3, 536-543.

5. Yi, P.; Poulikakos, D.; Walther, J.; Yadigaroglu, G. Molecular dynamics simulation of vaporization of an ultra-thin liquid Argon layer on a surface. International Journal of Heat and Mass Transfer, 2002, Vol. 45, No. 10, pp. 2087-2100.

6. Wang, Z. J.; Chen, M.; Guo, Z. Y. A non-equilibrium molecular dynamics simulation of evaporation. International Conference Passive and Low Energy Cooling for the Built Environment, 2005, Santorini, Greece Vol.1, pp. 543-547.

7. Toghraie Semiromi, D.; Azimian, A. R. Molecular dynamics simulation of annular flow boiling with the modified Lennard-Jones potential function. Heat and Mass Transfer, 2012, Vol. 48, pp. 141-152.

8. Morshed, A.K. M.M.; Paul, T. C.; Khan, J. A. Effect of nanostructures on evaporation and explosive boiling of thin liquid films: a molecular dynamics study. Applied Physics A Materials Science $\mathcal{E}$ Processing, 2011, Vol. 105, pp. 445-451. 
9. Toghraie Semiromi, D.; Azimian, A. R. Molecular dynamics simulation of liquid-vapor phase equilibrium by using the modified Lennard-Jones potential function. Heat and Mass Transfer, 2010, Vol. 46, pp. 287-294.

10. Stoddard, S. D.; Ford, P. J. Numerical experiments on the stochastic behavior of a Lennard-Jones gas system. Physical Review A, 1973, Vol. 8, pp. 1504-1512.

11. Thompson, P. A.; Robbins, A. O. Shear flow near solids: Epitaxial order and flow boundary conditions. Physical Review A, 1990, Vol. 41, pp. 6830-6837.

12. Berne, B. J.; Hua, L.; Zangi, R. Hydrophobic interactions and dewetting between plates with hydrophobic and hydrophilic domains. Annual Review of Physical Chemistry, 2009, Vol. 113, pp. 5244-5253.

13. Tuteja, A.; Choi, W.; Ma, M.; Mabry, J. M.; Mazzella, S. A.; Rutledge, G. C.; McKinley, G. H.; Cohen, R. E. Designing Superoleophobic Surfaces. Science, 2007, Vol. 318, Issue 5856, pp. 1618-1622.

14. Jabbarzadeh, A. Effect of nano-patterning on oleophobic properties of a surface. Soft Matter, 2013, Vol. 9, pp. 11598-11608.

15. Sedighi, N.; Murad, S.; Aggarwal, S. K. Molecular dynamics simulations of nanodroplet spreading on solid surfaces, effect of droplet size. Fluid Dynamics Research, 2010, Vol. 42, pp. 1-23.

16. Yi, P.; Poulikakos, D.; Walther, J.; Yadigaroglu, G. Molecular dynamics simulation of vaporization of an ultra-thin liquid Argon layer on a surface. International Journal of Heat and Mass Transfer, 2002, Vol. 45, pp. 2087-2100.

17. Ji, C. Y.; Tsuruta, T.; Nagayama, G. Effects of solid heating surface on nanometer sized liquid films. Thermal Science and Engineering, 2005, Vol. 13, pp. 25-28.

18. Sutmann, G. Classical molecular dynamics. John Von Neumann Institute for Computing, Jülich, NIC Series, 2002, Vol.10, pp. 211-254.

19. Verlet, L. Computer "Experiments" on Classical Fluids. I. Thermodynamical Properties of Lennard-Jones Molecules. Physics Review, 1976, pp. 159-198.

20. Alexander, F. J.; Garcia, A. L. The direct simulation of Monte-Carlo method. Computer Simulation Journal, 1997, Vol. 11, pp.588-593.

21. Xu, J. L.; Zhou, F. J.; Garcia, A. L. Molecular dynamics simulation of micro- Poiseuille flow for liquid argon in nanoscale. Journal of Heat and Mass Transfer, 2004, Vol. 47, pp. 501-513.

22. Haile, J. M. Molecular dynamics simulation, elementary methods. Wiley-Interscience Publication, 1997.

23. Nagayama, G.; Cheng, P. Effects of interface wettability on microscale flow by molecular dynamics simulation. International Journal of Heat and Mass Transfer, 2004, Vol. 47, pp. 501-513.

24. Zhigilei, L. V.; Kodali, P. B. S.; Garrison, B. J. Molecular Dynamics Model for Laser Ablation of Organic Solids. The Journal of Physical Chemistry B, 1999, Vol. 101, No. 11, pp. 2028-2037.

(C) 2016 by the authors; licensee Preprints, Basel, Switzerland. This article is an open access article distributed under the terms and conditions of the Creative Commons by Attribution (CC-BY) license (http://creativecommons.org/licenses/by/4.0/). 